\title{
AUTOEFICACIA ESPECÍFICA PARA LA JUBILACIÓN, INTENCIONES DE RETIRO Y SATISFACCIÓN VITAL EN TRABAJADORES MAYORES DE 40 AÑOS
}

\author{
RETIREMENT SELF EFFICACY, \\ RETIREMENT INTENTIONS AND \\ LIFE SATISFACTION AMONG \\ WORKERS AGED 40 AND OVER
}

\author{
ENCARNA VALERo IbÁÑEZ ${ }^{1}$, AdRIÁN SEGURA ${ }^{1,2}$ \\ Y GABRIELA TOPA CANTISANO ${ }^{2}$
}

\begin{abstract}
Cómo referenciar este artículo/How to reference this article:
Valero Ibáñez, E., Segura, A. y Topa Cantisano, G. (2015). Autoeficacia específica para la Jubilación, intenciones de retiro y satisfacción vital en trabajadores mayores de 40 años [Retirement self efficacy, retirement intentions and life satisfaction among workers aged 40 and over]. Acción Psicológica, 12(1), 35-46. doi: http://dx.doi.org/10.5944/ap.12.1.14897
\end{abstract}

\section{Resumen}

La autoeficacia para la jubilación incluye las percepciones de la medida en la cual uno será capaz de enfrentarse con las tareas que implica la nueva situación de retiro. Con esta investigación se exploran los factores psicosociales que pronostican las intenciones de retiro y la satisfacción con la vida cerca de la jubilación. El estudio se ha llevado a cabo con una muestra de 1,325 trabajadores españoles mayores. La autoeficacia para la jubilación pronostica las intenciones de retiro (jubilación parcial y total) y la satisfacción vital cercana a la jubilación, añadiendo poder predictivo a la autoeficacia generalizada. Pese a este hecho, solo dos dimensiones de la autoeficacia para la jubilación explican exitosamente todos los resultados (las finanzas y la salud). Nuestros hallazgos subrayan que la autoeficacia para la jubilación pronostica positivamente las intenciones de jubilación total y la satisfacción con la jubilación de los trabajadores mayores, y negativamente las intenciones de jubilación parcial. Las implicaciones de estos estudios se discuten de cara a entender la planificación de la jubilación y a mejorar las prácticas de orientación a las personas mayores.

Palabras clave: jubilación; satisfacción vital; trayectorias; bienestar.

Correspondencia: Gabriela Topa Cantisano. Universidad Nacional de Educación a Distancia (UNED).

Email: gtopa@psi.uned.es

${ }^{1}$ Universidad de Huelva.

${ }^{2}$ Universidad Nacional de Educación a Distancia (UNED).

Recibido: 23 de febrero de 2015

Aceptado: 24 de mayo de 2015 


\begin{abstract}
Retirement self-efficacy includes perceptions of the extent to which one will be capable of dealing with the tasks involved in the new retiree situation. This research aims to explore the psychosocial factors that predict retirement intentions and satisfaction with life near retirement. The study has been conducted with a sample of 1,325 Spanish workers aged 40 and over. Retirement selfefficacy predicted retirement intentions (part-time retirement, and full retirement) and life satisfaction near retirement, adding predictive power to generalized self efficacy. Despite this fact, only two Retirement self efficacy dimensions successfully explained all the results (finances and health). Our findings highlight that retirement self efficacy positively predict both full retirement and retirement satisfaction of aged workers, and negatively predicted partial retirement intentions. The implications of these studies are discussed for understanding retirement planning and counselling practice.
\end{abstract}

Keywords: retirement; retirement satisfaction; trajectories; wellbeing.

\section{Introducción}

La jubilación es un acontecimiento clave en la vida de las personas debido a que supone una transición, casi siempre irreversible, desde la situación laboral activa a la situación de retiro. Los trabajadores que se acercan a la jubilación tienen que decidir entre los posibles itinerarios de salida del mercado de trabajo. Además, deben tomar conciencia de las tareas que están implicadas, tanto en el paso hacia esta nueva situación como en la gestión eficaz de la nueva vida como jubilados (Alcover, Topa y Fernández, 2014). Estas tareas implicadas en la transición son variadas y afectan a aspectos muy importantes de la vida personal y social, tales como la salud, las finanzas, las actividades y relaciones sociales, entre otras (Shultz y Wang, 2007). En este sentido, un constructo de especial importancia, es la Autoeficacia Especifica para la Jubilación (en adelante, AEJ).

La autoeficacia en general se refiere a aquello que piensan y sienten las personas respecto a su capacidad para llevar a cabo tareas específicas bajo una gran variedad de circunstancias alcanzando un nivel de rendimiento determinado (Bandura, 1997). Por AEJ, por tanto, se entienden las creencias de las personas sobre su potencial eficacia para resolver los desafíos que implica la jubilación (Neuhs, 1990; Harper, 2005). El impacto de la autoeficacia es amplio, puesto que afecta a las metas y aspiraciones, las motivaciones para intentar una tarea, la persistencia, el rendimiento, el éxito, el estrés relacionado con las tareas y la satisfacción global, como ponen de manifiesto gran cantidad de estudios empíricos y varias revisiones meta-analíticas (Stajovic y Luthans, 1998).

Algunos estudios han señalado que los hallazgos sobre autoeficacia en otros contextos se aplican a la jubilación (Fretz, Kluge, Ossana, Jones y Merikangas, 1989; Taylor y Shore, 1995). Los trabajadores con mayor AEJ tienden a planificar su retiro anticipadamente y experimentan menos ansiedad frente a la jubilación. En relación con el proceso de retiro, la AEJ es probable que afecte a las intenciones de retiro de los trabajadores (Taylor y Shore, 1995), a sus sentimientos acerca del futuro retiro (Fretz et al., 1989), y a la motivación para afrontar la transición (Taylor y Shore, 1995). La investigación específica sobre la AEJ aún es limitada, tanto por el desarrollo de los instrumentos de medida como por el alcance de sus estudios empíricos.

La mayoría de las investigaciones que incluyen la autoeficacia para la jubilación han usado medidas monoítem o escalas muy reducidas y sin adecuado análisis psicométrico. Estudios realizados con una escala más extensa de autoeficacia para la jubilación han mostrado correlaciones positivas con la satisfacción vital, y negativas con la ansiedad frente a la jubilación (Neuhs, 1990). Finalmente, Harper (2005) ha desarrollado una escala de AEJ, llamada Retirement Questionnaire, modificando y actualizando la versión original de Neuhs, que ha mostrado adecuadas propiedades psicométricas. Esta escala incluye una amplia taxonomía de tareas implicadas en el retiro, tales como el cuidado de la salud física y mental, la gestión financiera, la organización de las actividades y relaciones sociales y un conjunto de decisiones de amplio alcance, tales como el lugar de residencia o el uso del tiempo libre (Harper, 2005). Sin embargo, en la mayoría de los estudios esta información no se aprovecha para análisis posteriores, porque se agregan las medidas (Peila-Shuster, 2011; Topa y Alcover, 2015). A pesar de que algunos de sus hallazgos eran prometedores, además tales estudios tenían limitada generalizabilidad a causa de que las muestras usadas no eran pertinentes, ya que incluían sólo a jóvenes universitarios. 


\section{Intenciones de retivo en la transición hacia la jubilación}

La decisión personal de retirarse está determinada por factores objetivos, tales como la edad, los años de servicio, los requisitos exigidos para disfrutar de una pensión suficiente, y por un conjunto de factores subjetivos, tales como la edad que se percibe como normativa para jubilarse en un ámbito profesional concreto, la imagen que las personas tengan de sí mismas como trabajadores, o la etapa de la carrera profesional que estén atravesando, entre otros muchos (Feldman, 2013).

Estos aspectos psicosociales pueden conducir a las personas a percibir que ha llegado el momento de alejarse del mundo laboral o, por el contrario, pueden alimentar su deseo de permanecer en él. Son diversas las causas por las que algunos de estos adultos que se podrían jubilar continúan trabajando, como por ejemplo por disfrute personal y porque el trabajo les proporciona significado, estructura, propósito a sus vidas, además de una red de relaciones sociales. Otras personas lo que buscan es mantener su estatus económico para suplir pensiones que son inadecuadas desde el punto de vista financiero. En cualquier caso, muchas de las personas readaptan su trabajo, reduciendo su jornada laboral o desempeñando tareas en forma parcial por las dos razones señaladas anteriormente $o$, simplemente, porque no se pueden permitir retirarse (Topa y Alcover, 2015; Zappalà, Depolo, Fraccaroli, Guglielmi y Sarchielli, 2008). Aunque la mayoría de la investigación precedente se ha centrado en perspectivas macro o micro económicas a la hora de pronosticar las intenciones de jubilación, en este estudio interesa explorar algunos predictores psicosociales de la intención de retiro parcial o de retiro total.

\section{La satisfacción vital durante la transición hacia lajubilación}

La satisfacción vital es una valoración global de la persona sobre su vida, que ésta hace basándose en sus propios criterios (Diener, Emmons, Larsen y Griffin, 1985). Sin embargo, algunos autores insisten en que las evaluaciones globales por sí solas no son capaces de dar cuenta de la calidad de vida en aspectos específicos y concretos de la vida personal (Campbell, Converse y Rodgers, 1976). Por este motivo, en el ámbito de la investigación sobre la jubilación, una de las escalas más difundidas ha sido la de Satisfacción con la vida en la jubilación, que incluye un conjunto de ítems evaluadores de la satisfacción con áreas específicas (Floyd, Haynes, Doll y Winermiller, 1992). Las áreas específicas incluidas en el constructo de satisfacción con la vida en la jubilación y en su medida son comunes con otras escalas de satisfacción vital, e incluyen los recursos económicos, las relaciones interpersonales con otras personas relevantes, la calidad de los servicios sociales y el acceso al transporte, entre otros aspectos.

Los estudios muestran que la satisfacción vital tiene relaciones negativas con la ansiedad acerca de la jubilación y relaciones positivas con la AEJ (Neuhs, 1990). Debido al escaso número de estudios, un examen más detallado de estas relaciones parece oportuno.

El presente estudio de tipo correlacional tiene por objeto la evaluación del AEJ y de sus relaciones con las intenciones de jubilación y con la satisfacción vital en la jubilación. Debido a que los estudios precedentes emplearon escalas muy breves y muestras no pertinentes, en este caso se utiliza un instrumento que incluye todas las dimensiones de la AEJ y una muestra amplia y diversa de trabajadores mayores.

Esto nos permitirá conocer, en primer lugar, si la AEJ es un predictor eficaz de las intenciones de retiro. En segundo lugar, nos permitirá establecer si efectivamente la AEJ añade algún poder predictivo sobre la satisfacción vital durante la transición desde el trabajo a la jubilación, por encima de la autoeficacia general.

\section{Método}

\section{Participantes}

Este estudio se ha llevado a cabo con 1.325 trabajadores cuya edad eran 40 años o más en el momento de participar y que se encontraban en situación laboral activa. El 56\% eran varones y la edad media de los participantes era 60.8 años $(S D=3.15)$. Los trabajadores residían en España y trabajaban por cuenta ajena. En relación con su distribución por regiones, el $32.8 \%$ pertenecían a la zona norte (País Vasco, Navarra, Cantabria y Galicia), el $28.6 \%$ a Castilla La Mancha y Andalucía, el $13.7 \%$ a la zona de Levante e islas (Castellón, Valencia, Murcia, Baleares y Canarias), $12.8 \%$ a la Región central de España (Madrid y Comunidad de Castilla León) y $12.1 \%$ a Extremadura.

La distribución por sectores de actividad de la muestra indica que $38.9 \%$ pertenecían al sector servicios, $16.8 \%$ a la sanidad, $19.5 \%$ a educación, $8.4 \%$ al comercio y atención directa al público, $7.2 \%$ a finanzas y el resto a la industria en general. La antigüedad media 
como trabajadores era 26.5 años $(\mathrm{SD}=6.9)$. Solamente el $34.5 \%$ de los participantes tenía estudios universitarios.

\section{Procedimiento}

Para contactar con los trabajadores que constituyeron la muestra se contó con la colaboración de directores de RRHH que participaban en un curso de formación continua de la UNED a través de la plataforma de eLearning. Los participantes fueron informados de los objetivos generales del estudio, del carácter anónimo del estudio, y del tratamiento global de sus respuestas únicamente para fines académicos, tras lo cual expresaron su intención de participar a través de un documento de consentimiento informado. Los participantes recibieron un sobre con el siguiente contenido: carta de presentación del estudio, escalas a cumplimentar, y sobre para guardar el cuestionario una vez cumplimentado. Los sobres con las encuestas fueron entregados al equipo investigador. Se distribuyeron inicialmente 2000 cuestionarios, de los que sólo se recogieron 1.567 (tasa de respuesta $78.5 \%$ ). Se eliminaron todos aquellos cuadernillos que contenían más del $25 \%$ de datos perdidos, con lo que se retuvo una muestra final de 1.325 participantes.

\section{Instrumentos de evaluación}

Autoeficacia Generalizada. Se utilizó la versión española de la Escala de Autoeficacia General (Schwarzer y Baessler, 1996), con diez ítems. Los estudios previos han mostrado una fiabilidad adecuada para esta versión que oscila entre $\alpha=.81$ (Schwarzer, Baessler, Kwiatek, Schroder y Zhang, 1997) y $\alpha=.83$ (Juárez y Contreras, 2008). En el presente estudio la fiabilidad fue de $\alpha=$ .92 .

AEJ. Se utilizó una versión traducida al español de la escala Retirement Questionnaire (Harper, 2005) que incluía 41 ítems en su forma original, con seis subescalas: salud física y mental, finanzas, actividades, gestiones ante la administración, pensiones y decisiones acerca de la jubilación. Este cuestionario se aplicó a un estudio piloto de 101 trabajadores mayores. El análisis de estos datos permitió retener sólo 24 ítems con cargas factoriales más altas en el Análisis Factorial Exploratorio (AFE). El análisis de contenido de los ítems excluidos confirmó que se trataban de las subescalas relativas agestiones ante la administración (siete ítems) y pensiones (siete ítems) y de tres ítems de la subescala de salud física cuyo contenido no era aplicable al contexto espa- ñol. Estos ítems estaban centrados en actividades tales como cumplimentación de formularios para la aseguradora médica, búsqueda de proveedores de seguros médicos, participación en seminarios formativos para el ahorro y otras actividades que son muy poco frecuentes entre los trabajadores mayores españoles. La escala de respuesta para que los participantes evaluasen su autoeficacia específica era del tipo Likert de cinco puntos y oscilaba entre 1 (Totalmente inseguro) a 5 (Totalmente seguro). La fiabilidad de las subescalas resulta adecuada, siendo $\alpha=$ .87 para la subescala de salud (seis ítems), $\alpha=.88$ para la subescala financiera (seis ítems), $\alpha=.85$ para la subescala decisiones acerca de la jubilación (seis ítems) y $\alpha=.88$ para la subescala de actividades (seis ítems). La escala completa se ofrece en el anexo.

Satisfacción vital en la jubilación. Se empleó una traducción al español de la subescala de satisfacción con la vida en la jubilación, que es parte del Retirement Satisfaction Inventory (RSI; Floyd et al., 1992) e incluye 11 ítems. Muchos de los aspectos que se incluyen son comunes a otras escalas de satisfacción vital (recursos financieros o relaciones interpersonales) y otros son particularmente relevantes para los futuros jubilados, tales como el acceso a los servicios sociales o al transporte. La fiabilidad por Alpha de Cronbach fue de $\alpha=82$ en el presente estudio para la escala global y $\alpha=.78$ en la subescala de satisfacción con la calidad de vida general (cinco ítems), $\alpha=.80$ en la subescala de satisfacción con el matrimonio y vida familiar (cuatro ítems) y $\alpha=.70$ en la subescala de satisfacción con la salud física, que contiene sólo dos ítems.

Intenciones de retiro. Se tradujo la escala de intenciones de transición hacia la jubilación total y hacia la jubilación parcial (Australian Bureau of Statistics, 2012). El estudio original no reporta medidas de fiabilidad, pero ambas subescalas mostraron consistencia interna adecuada en el presente trabajo, con $\alpha=.83$ en la subescala de intenciones de jubilación parcial (cinco ítems) y $\alpha=.79$ en la subescala de intenciones de jubilación total (tres ítems).

Datos sociodemográficas. Se han evaluado la edad y la antigüedad en el empleo de los trabajadores, y estas variables se han controlado en los análisis de regresión sobre la base de la literatura (Gallo, Bradley, Siegel y Kasl, 2001). 
Tabla 1

Matriz de correlaciones y estadísticos descriptivos de las variables del Estudio $1(N=1.325)$

\begin{tabular}{|c|c|c|c|c|c|c|c|c|c|c|c|c|c|}
\hline Variables & $M$ & $D T$ & 1 & 2 & 3 & 4 & 5 & 6 & 7 & 8 & 9 & 10 & 11 \\
\hline 1. Edad & 60.8 & 3.2 & - & & & & & & & & & & \\
\hline 2.Antiguedad empleo & 26.5 & 6.9 & $.26^{* *}$ & - & & & & & & & & & \\
\hline 3. Autoeficacia general & 3.4 & .62 & .04 & $.07^{*}$ & - & & & & & & & & \\
\hline 4. RSE salud & 3.9 & .69 & -.02 & .03 & $.37^{* *}$ & - & & & & & & & \\
\hline 5. RSE finanzas & 3.4 & .80 & -.04 & $.06^{*}$ & $.31^{* *}$ & $.40^{\star *}$ & - & & & & & & \\
\hline 6. RSE actividades & 3.7 & .76 & -.02 & .00 & $.40^{\star *}$ & $.50^{* *}$ & $.39^{* *}$ & - & & & & & \\
\hline 7. RSE decisiones & 3.67 & .75 & -.00 & .00 & $.38^{* *}$ & $.47^{* *}$ & $.61^{* *}$ & $.51^{* *}$ & - & & & & \\
\hline $\begin{array}{l}\text { 8. RSI servicios y recur- } \\
\text { sos }\end{array}$ & 3.18 & .74 & -.00 & $.06^{*}$ & $.35^{\star \star}$ & $.29^{* *}$ & $.47^{* *}$ & $.32^{\star *}$ & $.43^{* *}$ & - & & & \\
\hline $\begin{array}{l}\text { 9. RSI matrimonio y vida } \\
\text { familiar }\end{array}$ & 3.90 & .74 & .00 & $.09^{* *}$ & $.35^{\star \star}$ & $.34^{* *}$ & $.33^{* *}$ & $.26^{\star \star}$ & $.37^{* \star}$ & $.42^{\star *}$ & - & & \\
\hline 10. RSI salud y actividad & 3.60 & .80 & -.01 & .06 & $.38^{* *}$ & $.38^{* *}$ & $.31^{* *}$ & $.37^{\star *}$ & $.32^{\star *}$ & $.45^{\star *}$ & $.41^{* *}$ & - & \\
\hline $\begin{array}{l}\text { 11. Intención retiro par- } \\
\text { cial }\end{array}$ & 1.80 & 1.21 & .02 & -.00 & $.11^{* *}$ & $.07^{* *}$ & -.02 & $.07^{\star-}$ & -.02 & -.00 & $.11^{* *}$ & .04 & - \\
\hline 12.Intención retiro total & 3.60 & 1.05 & $.06^{*}$ & $.07^{*}$ & $.28^{* *}$ & $.18^{* *}$ & $.22^{* *}$ & $.19^{* *}$ & $.18^{* *}$ & $.22^{* *}$ & $.20^{* *}$ & $.19^{* *}$ & $.13^{* *}$ \\
\hline
\end{tabular}

Nota. RSE: Retirement self-efficacy, autoeficacia específica para la jubilación; RSI: Retirement satisfaction inventory, satisfacción con la jubilación; M: media; DT: desviación típica.

\section{Análisis de datos}

Con objeto de poner a prueba la estabilidad de la estructura de la diferentes dimensiones de las escalas de AEJ y de Satisfacción vital en la jubilación, éstas se sometieron a sendos Análisis factoriales confirmatorios (AFC), con el programa Amos 19.0. En ambos casos se empleó el procedimiento de máxima verosimilitud y se valoraron los indicadores de ajuste del modelo recomendados, tales como el Goodness of Fit Index (GFI), el Adjusted Goodness of Fit Index (AGFI), el Comparative Fit Index (CFI) que deben alcanzar valores superiores .90. Además, el Standardized Root Mean Square Residual (SRMR), y el Root Mean Squared Error of Approximation (RMSEA), que deben ser inferiores a .08 , mientras el valor de $\square /$ d.f. debe ser inferior a 4.0.

En la escala de Autoeficacia para la jubilación, el modelo incorporó 24 variables endógenas y 28 variables exógenas. Todos los resultados cumplen los requerimientos como para concluir que el modelo de cuatro factores muestra un ajuste aceptable $\square=814.3$, d.f. $=$ 234, $\square$ /d.f. $=3.4$, GFI $=.95$, AGFI $=.93, \mathrm{CFI}=.97$, RMR $=.03$, RMSEA $=.03$ ). Todos los ítems muestran cargas factoriales superiores a .50 en su correspondiente factor y ningún ítem se relaciona con más de un factor, pese a lo cual el ajuste del modelo es mejorable.

En la escala de Satisfacción vital en la jubilación, el modelo empleado en el AFC 11 variables endógenas y 14 exógenas. Los resultados cumplen los requerimientos para considerar que el modelo de tres factores tiene un ajuste aceptable $[\square=109.6$, d.f. $=33, \square /$ d.f. $=3.3$,
$\mathrm{GFI}=.99, \mathrm{AGFI}=.97, \mathrm{CFI}=.98, \mathrm{RMR}=.03, \mathrm{RMSEA}$ $=.04]$. Los ítems presentan, en su mayoría, cargas factoriales superiores a .50 en el factor correspondiente y ningún ítem se relaciona con más de un factor.

\section{Resultados}

\section{Análisis descriptivos y correlaciónales}

La Tabla 1 muestra los estadísticos descriptivos y las correlaciones entre las variables del estudio. Los resultados revelan que las intenciones de jubilación total son altas, mientras que las de jubilación parcial son bajas y con alta variabilidad. Entre los datos sociodemográficos, la edad sólo muestra relaciones significativas con la antigüedad en el empleo $(r=.26)$ y con la intención de jubilación total $(r=.06)$. La antigüedad en el empleo se relaciona de forma estadísticamente significativa con la satisfacción vital en la jubilación en sus tres dimensiones y con la intención de retiro completo $(r=.28)$. Las cuatro dimensiones de la autoeficacia específica se relacionan de modo estadísticamente significativo con la autoeficacia general y con la satisfacción vital en la jubilación. En cuanto a las intenciones de jubilación total, todas las dimensiones de autoeficacia para la jubilación tienen relaciones significativas, mientras que para las intenciones de retiro parcial, solo las dimensiones de autoeficacia para la salud y para las actividades muestran relación estadísticamente significativa, siendo además sus coeficientes de signo negativo $(r=-.07)$. 
Tabla 2

Análisis de Regresión Lineal Múltiple para satisfacción con servicios y recursos

\begin{tabular}{|c|c|c|c|}
\hline & \multicolumn{3}{|c|}{ Satisfacción con los servicios y recursos en la jubilación } \\
\hline & \multicolumn{3}{|c|}{ Modelo } \\
\hline & 1 & 2 & 3 \\
\hline Variables predictoras & $\beta^{\mathrm{a}}$ & $\beta^{a}$ & $\beta^{\text {a }}$ \\
\hline Edad & -.02 & -.03 & -.00 \\
\hline Antigüedad en el empleo & .05 & .02 & .02 \\
\hline Autoeficacia general & & $.35^{* * *}$ & $.17^{* * *}$ \\
\hline RSE finanzas & & & $.30^{* * *}$ \\
\hline RSE decisiones & & & $.17^{* \star *}$ \\
\hline$R^{2}$ & .002 & .12 & .30 \\
\hline$\Delta R^{2}$ & .002 & $.12^{* \star *}$ & $.18^{* * *}$ \\
\hline$F$ & 1.36 & $51.9^{* *}$ & $67.11^{* *}$ \\
\hline$\Delta F$ & 1.36 & $152.7^{* * *}$ & $68.9^{* * *}$ \\
\hline
\end{tabular}

Nota. a Coeficientes estandarizados beta; RSE: Retirement self-efficacy, autoeficacia específica para la jubilación. ${ }^{*} p$ $<.05 ;{ }^{* *} p<.01 ;{ }^{* \star *} p<.001$.

\section{Eficacia predictiva de la AEJ sobre las intenciones de retiro de los trabajadores mayores}

El análisis de regresión lineal múltiple, con el procedimiento Stepwise (por pasos), se utiliza para poner a prueba las hipótesis del estudio. Este procedimiento permite hallar el modelo más parsimonioso, con el número más adecuado de variables independientes, no redundantes. La decisión de excluir variables se basa en criterios estadísticos, eliminando aquellas que no contribuyen de forma significativa a la explicación de varianza adicional (López, 1998). En relación con la predicción de la satisfacción con los servicios y recursos en la jubilación (Hipótesis 1a), en el primer modelo se incluyen sólo las variables sociodemográficas, edad y tiempo en el empleo, de las que ninguna resulta ser predictor significativo, dando este modelo cuenta de una parte ínfima de la varianza de la variable criterio. En el segundo modelo, la autoeficacia general es un potente predictor de la satisfacción, aumentando así el porcenta- je de varianza explicada que pasa al $12.1 \%$. Finalmente, el tercer modelo el porcentaje de varianza explicada pasa al $30 \%$, con un poder predictivo sobre la satisfacción con los servicios y recursos en la jubilación de las dimensiones de la autoeficacia específica para la jubilación referidas a las finanzas y a la toma de decisiones acerca de la jubilación (ver Tabla 2).

En relación con la predicción de la satisfacción con el matrimonio y la vida familiar (Hipótesis 1b), en el primer modelo se incluyen sólo las variables sociodemográficas, edad y tiempo en el empleo, de las que la segunda resulta ser predictor significativo, dando este modelo cuenta de una parte ínfima de la varianza de la variable criterio. En el segundo modelo, la autoeficacia general es un potente predictor de la satisfacción con el matrimonio y la vida familiar, aumentando así el porcentaje de varianza explicada que pasa al $12.3 \%$. Finalmente, el tercer modelo el porcentaje de varianza explicada pasa al $21 \%$, con un poder predictivo sobre la satisfacción con el matrimonio y la vida familiar de las dimensiones de la autoeficacia

Tabla 3

Análisis de Regresión Lineal Múltiple para satisfacción con matrimonio y vida familiar

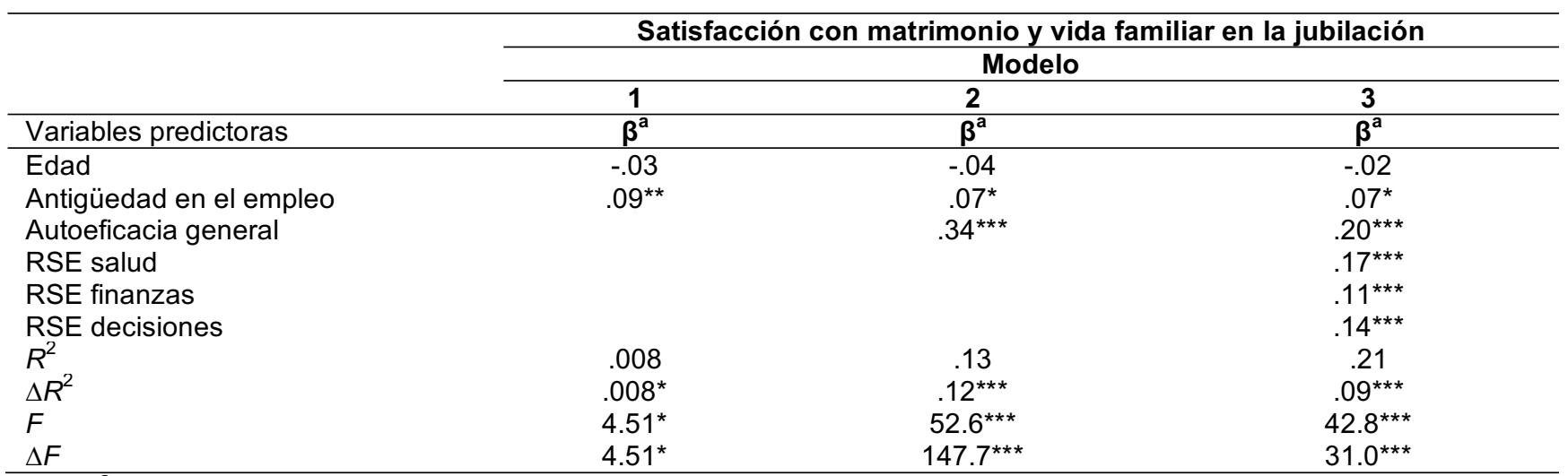

Nota. ${ }^{a}$ Coeficientes estandarizados beta; RSE: Retirement self-efficacy, autoeficacia específica para la jubilación. 
Análisis de Regresión Lineal Múltiple para satisfacción con salud y actividad

\begin{tabular}{|c|c|c|c|}
\hline & \multicolumn{3}{|c|}{ Satisfacción con salud y actividad en la jubilación } \\
\hline & \multicolumn{3}{|c|}{ Modelo } \\
\hline & 1 & 2 & 3 \\
\hline Variables predictoras & $\beta^{\mathrm{a}}$ & $\beta^{\mathrm{a}}$ & $\beta^{a}$ \\
\hline Edad & -.03 & -.04 & -.02 \\
\hline Antigüedad en el empleo & .05 & .03 & .03 \\
\hline Autoeficacia general & & $.38^{* * *}$ & $.21^{* * *}$ \\
\hline RSE salud & & & $.20^{* * *}$ \\
\hline RSE finanzas & & & $.14^{* * *}$ \\
\hline RSE actividades & & & $.15^{* * *}$ \\
\hline$R^{2}$ & .003 & .15 & .26 \\
\hline$\Delta R^{2}$ & .003 & $.14^{* * *}$ & $.11^{* * *}$ \\
\hline$F$ & 1.50 & $63.11^{* * *}$ & $54.8^{* * *}$ \\
\hline$\Delta F$ & 1.50 & $185.6^{\star \star *}$ & $41.5^{* * *}$ \\
\hline
\end{tabular}

Nota. ${ }^{a}$ Coeficientes estandarizados beta; RSE: Retirement self-efficacy, autoeficacia específica para la jubilación. ${ }^{*} p<.05 ;{ }^{* *} p<.01 ;{ }^{* * *} p<.001$.

específica para la jubilación referidas a la salud, a las finanzas y a la toma de decisiones acerca de la jubilación (ver Tabla 3).

En relación con la predicción de la satisfacción con la salud y la actividad en la jubilación (Hipótesis 1c), en el primer modelo se incluyen sólo las variables sociodemográficas, edad y tiempo en el empleo, de las que ninguna es significativa. El porcentaje de varianza explicado es prácticamente nulo. En el segundo modelo, la autoeficacia general es predictora de la satisfacción con la salud y la actividad en la jubilación, aumentando así el porcentaje de varianza explicada que pasa al $14.4 \%$. Finalmente, el tercer modelo el porcentaje de varianza explicada pasa al $25 \%$, con un poder predictivo sobre la satisfacción con la salud y la actividad en la jubilación de todas las dimensiones de la autoeficacia específica para la jubilación, con excepción de la capacidad para

Tabla 5

Análisis de Regresión Lineal Múltiple para intención de jubilación parcial

Satisfacción con salud y actividad en la jubilación

\begin{tabular}{|c|c|c|c|c|c|}
\hline & \multicolumn{5}{|c|}{ Modelo } \\
\hline & 1 & 2 & 3 & 4 & 5 \\
\hline Variables predictoras & $\beta^{a}$ & $\beta^{a}$ & $\beta^{a}$ & $\beta^{a}$ & $\beta^{a}$ \\
\hline Edad & .00 & .00 & -.00 & .00 & -.004 \\
\hline Antigüedad en el empleo & -.02 & -.03 & -.03 & -.02 & -.02 \\
\hline Autoeficacia general & & $.11^{\star \star *}$ & $.17^{\star \star *}$ & $.14^{\star \star *}$ & $.15^{\star * *}$ \\
\hline RSE salud & & & $-.16^{* * *}$ & $-.20 * * *$ & $-.18^{* * *}$ \\
\hline RSE actividades & & & & $-.11^{* *}$ & $-.12^{* \star}$ \\
\hline RSE finanzas & & & & & $-.07^{*}$ \\
\hline$R^{2}$ & .00 & .01 & .035 & .042 & .046 \\
\hline$\Delta R^{2}$ & .00 & $.01^{* * *}$ & $.02^{* * *}$ & $.007^{* *}$ & $.004^{*}$ \\
\hline$F$ & .27 & $4.65^{\star *}$ & $9.9^{* * *}$ & $9.6^{* * *}$ & $8.8^{* * *}$ \\
\hline$\Delta F$ & .27 & $13.4^{* * *}$ & $25.2^{\star \star *}$ & $8.08^{* *}$ & $4.8^{*}$ \\
\hline
\end{tabular}

Nota. ${ }^{a}$ Coeficientes estandarizados beta; RSE: Retirement self-efficacy, autoeficacia específica para la jubilación. ${ }^{*} p<.05 ;{ }^{* *} p<.01 ;{ }^{* * *} p<.001$. 
Tabla 6

Análisis de Regresión Lineal Múltiple para intención de retiro total

\begin{tabular}{|c|c|c|c|}
\hline & \multicolumn{3}{|c|}{ Satisfacción con salud y actividad en la jubilación } \\
\hline & \multicolumn{3}{|c|}{ Modelo } \\
\hline & 1 & 2 & 3 \\
\hline Variables predictoras & $\beta^{a}$ & $\beta^{a}$ & $\beta^{a}$ \\
\hline Edad & .06 & .05 & .06 \\
\hline Antigüedad en el empleo & .05 & .04 & .03 \\
\hline Autoeficacia general & & $.28^{* * *}$ & $.23^{* * *}$ \\
\hline RSE finanzas & & & $.16^{* * *}$ \\
\hline$R^{2}$ & $.007^{*}$ & $.08^{* * *}$ & $.11^{\star * *}$ \\
\hline$\Delta R^{2}$ & $.007^{*}$ & $.08^{* * *}$ & $.02^{* \star *}$ \\
\hline$F$ & $4.07^{*}$ & $33.6^{\star * *}$ & $32.8^{* * *}$ \\
\hline$\Delta F$ & $4.07^{\star}$ & $92.02^{* \star *}$ & $28.0^{* \star *}$ \\
\hline
\end{tabular}

Nota. ${ }^{a}$ Coeficientes estandarizados beta; RSE: Retirement self-efficacy, autoeficacia específica para la jubilación. ${ }^{*} p$ $<.05 ;{ }^{* *} p<.01 ;{ }^{* * *} p<.001$.

Por último, los datos de Tabla 6 reflejan que la Hipótesis 3 del estudio resulta sólo parcialmente apoyada por los datos. En el Modelo 1 se observa que las variables sociodemográficas alcanzan valores de significación sólo tendenciales cuando pronostican la intención de jubilación total, siendo además el porcentaje de varianza explicada prácticamente irrelevante. En el Modelo 2 se observa que la autoeficacia general es un predictor eficaz de la intención de jubilarse totalmente, puesto que el nivel de probabilidad asociado al estadístico de contraste es significativo. En el Modelo 3, sólo la dimensión de manejo de finanzas de la autoeficacia específica para la jubilación muestra poder predictivo sobre la intención de jubilarse de forma total, y el porcentaje de varianza explicada por el modelo completo es superior al $10 \%$.

\section{Discusión}

El presente estudio tenía como objetivo verificar las relaciones entre la autoeficacia específica para la jubilación - de una parte- y las intenciones de retiro y la satisfacción vital con la vida cercana a la jubilación - de la otra parte. El estudio se llevó a cabo con una muestra amplia y heterogénea, en la cual tenían representación diversos sectores profesionales y regiones, y los resultados generales apoyan el modelo propuesto. Los hallazgos ponen de manifiesto que se ha cumplido el objetivo, aunque también suscitan nuevas preguntas de investigación para trabajos venideros.

En primer lugar, el presente trabajo supone un paso más en la línea incipiente de investigación sobre AEJ. Pese a que había algunos estudios previos (Fretz et al., 1989; Neuhs, 1990; Taylor y Shore, 1995), sus instrumentos de medida eran muy simples o sus muestras eran pequeñas, formadas por estudiantes o procedían de una única organización. Los hallazgos del presente trabajo se han basado una escala de AEJ, que cubre un abanico amplio de tareas, a las cuales han de enfrentarse las personas que se retiren. Esta escala además ha permitido hacer valoraciones de la autoeficacia por dimensiones, que no se habían dado, hasta donde sabemos, en los estudios anteriores, ya que incluso aquellos que la midieron por dimensiones, como Harper, (2005) o Topa y Alcover (2015), luego sumaban las referidas dimensiones para obtener una medida global de autoeficacia específica, con la consiguiente pérdida de información.

En segundo lugar, la AEJ para las finanzas emerge como la dimensión clave en relación con las intenciones de jubilación parcial y total. En la jubilación total, es la única dimensión de la autoeficacia con poder predictivo. Este hallazgo parece relevante, porque podría estar indicando que, más allá de todas las otras influencias de tipo actitudinal, la economía conserva un peso muy importante en las decisiones concretas relativas a la jubilación. En cuanto a la jubilación parcial, el peso de la AEJ para las finanzas es menor al de la AEJ para manejar la salud y la AEJ para manejar las actividades. Este resultado es consistente con otros hallazgos (Zaniboni, Sarchielli y Fraccaroli, 2010) que encuentra que el manejo de las relaciones sociales, o mejor dicho, el temor a perderlas es un predictor de la intención de retiro a tiempo parcial. También es consistente con los resultados precedentes de Topa y Alcover (2015), que encuentran un patrón de relaciones negativo de la autoeficacia con la intención de retiro parcial y positivo con la jubilación total, al igual que sucede en este estudio.

Por otra parte, ambos modelos predictivos de las intenciones de retiro, tanto parcial como total, son significativos en todos sus pasos pero poco eficientes, puesto que la varianza final que se explica de las intenciones es muy poca. Resultados semejantes se han encontrado en otros estudios, ya que Zaniboni y sus colegas (2010) pronostican las intenciones de retiro total y de retiro par- 
cial y encuentran que los pesos de regresión de sus predictores actitudinales son bajos, aunque resulten estadísticamente significativos. Igualmente sucede en el estudio precedente de Topa y Alcover (2015), dado que su modelo estructural no alcanza a pronosticar varianza de las intenciones de retiro más allá de un $25 \%$. Este hecho podría estar reflejando las múltiples influencias que se presentan sobre las decisiones de jubilarse o continuar retrasando la salida del mundo laboral. De hecho, los modelos teóricos más recientes que intentan dar cuenta de la jubilación anticipada hacen referencia, cuando menos, a cuatro planos diferentes en los que podría valorarse el ajuste entre la persona y el ambiente cuando se acerca la jubilación, el ajuste con la ocupación, con la organización, el puesto y el grupo de trabajo (Feldman, 2013; Oh et al., 2013). Visto desde esta perspectiva parece razonable que atender sólo a una faceta de las creencias de las personas nos devuelva un modelo con escasos porcentajes de varianza explicada. Quizá por esto la investigación precedente sobre los planes de retiro no ha logrado diferenciar claramente entre las diversas intenciones que las personas tienen al final de sus carreras laborales (Potočnik, Tordera y Peiró 2009).

En tercer lugar, en este estudio se pone de manifiesto que la autoeficacia general tiene un poder predictivo muy importante sobre las diferentes dimensiones de la satisfacción con la vida cercana a la jubilación. Así, se refuerzan los hallazgos de los estudios anteriores (Harper, 2005; Neuhs, 1990). Además, se hace un aporte adicional en el presente trabajo, ya que tales estudios evaluaron la AEJ, pero no mostraron que ésta tuviera poder predictivo por encima de la autoeficacia generalizada de la persona. En esta investigación siempre se ha introducido en los modelos de regresión la autoeficacia generalizada antes de la específica, a fin de controlar su influencia, tal como han recomendado estudios de autoeficacia en otros contextos (Salanova, Peiró y Schaufeli, 2002).

Sin embargo, este poder predictivo de las dimensiones de la AEJ, una vez se ha controlado la autoeficacia general, no se evidencia para todas las dimensiones sino sólo para algunas de ellas. En concreto, en la satisfacción con los servicios y recursos pesa más la autoeficacia para las finanzas, mientras que en la satisfacción con la vida familiar y en la satisfacción con la salud pesa más la autoeficacia para la salud. Así, se puede observar que hay relaciones estrechas entre la satisfacción y la AEJ, pero dichas relaciones podrían estar influidas por el contenido de los ítems de las diversas dimensiones, de una parte y de la otra.

Dado que en la presente investigación ambas medidas se han recogido en el mismo momento temporal y los datos proceden de la evaluación de una única fuente es previsible que pueda existir una alta intercorrelación entre tales subdimensiones. Efectivamente, al examinar la matriz de correlaciones ya se indicó la existencia de interrelaciones elevadas de la AEJ para la salud con la satisfacción con la salud $(r=.38)$, con la satisfacción con el matrimonio y la familia $(r=.33)$, y de la AEJ para controlar las finanzas con la satisfacción con los servicios y recursos $(r=.47)$.

En relación con este análisis también es destacable el hecho de que la AEJ para las finanzas sea un predictor eficaz de la satisfacción con la vida en todas sus dimensiones, es decir con independencia de qué dimensión concreta de la satisfacción se esté intentando explicar. Este hallazgo reafirma la profusa línea de investigación respecto a la importancia de las dimensiones económicas en la jubilación, resumida en diversos meta-análisis (Topa, Moriano, Depolo, Alcover y Morales, 2009; Topa, Moriano, Depolo, Alcover y Moreno, 2011). Por otra parte, avala también el peso de las medidas subjetivas de adecuación de los ingresos, que son reflejos de la preocupación de las personas por su bienestar económico cuando ya no puedan continuar ganando dinero por su trabajo (Taylor y Doverspike, 2003; Taylor y Gelhauser, 2007, Topa et al., 2011).

En esta misma línea, resulta destacable que la AEJ para la salud explica eficazmente dos de las tres dimensiones de la satisfacción vital. Este hecho vuelve a poner el acento en la importancia que tiene la salud, junto con la economía, en la preocupación de las personas cuando preparan su retiro (Shultz y Wang, 2007).

Ahora bien, las relaciones entre ingresos económicos y salud son muy intrincadas, a lo largo de todo el ciclo vital, y esto parece emerger también en la etapa de preparación de la jubilación y de jubilación. El déficit en salud previo impone limitaciones a las personas para alcanzar y conservar buenos empleos, y por consiguiente para ahorrar suficiente dinero para la jubilación (Himmelstein, Warren, Thorne, y Woolhandler, 2005). Además los ingresos bajos limitan el acceso de las personas a tratamientos sanitarios costosos y por consiguiente, les impiden disfrutar de una mejor calidad de vida (De Judicibus y McCabe, 2005; Hanratty, Holland, Jacoby y Whitehead, 2007), lo cual vuelve a poner de manifiesto la circularidad de las relaciones entre salud y finanzas.

Pese a sus aportes, este estudio tiene algunas limitaciones importantes que no se deben desatender. Por una parte, en relación con la representatividad de la muestra, las limitaciones del estudio son obvias, en especial debido al procedimiento de muestreo empleado. Aunque la muestra es amplia y heterogénea, no es representativa y esto impide hacer generalizaciones sólidas al resto de la 
población trabajadora del mismo segmento de edad. Además, el carácter autoinformada de los datos, que han sido evaluadas todas por la misma fuente, lleva a considerar el sesgo de varianza común como una fuente de amenaza a tener en cuenta. La validación de estos resultados a través de criterios independientes, como medidas objetivas de salud o respuestas de los familiares y amigos podrían ser usados en los estudios venideros. No obstante, dado que las medidas de autoeficacia están centradas en las expectativas subjetivas acerca del retiro, las desviaciones respecto a criterios externos no tienen que indicar necesariamente que se estén haciendo evaluaciones inadecuadas. En este mismo sentido, las medidas de AEJ podrían estar revelando un sesgo de optimismo ilusorio de las personas mayores respecto a la jubilación, el cual a su vez sería una ocasión para la intervención.

Los resultados de este estudio tienen implicaciones importantes de cara a la intervención con las personas mayores. Una vez mostrado el impacto de la AEJ en la satisfacción vital, sólo cabe señalar que los programas de preparación de la jubilación para las personas mayores deberían extenderse en número y centrarse en el desarrollo de estas creencias de autoeficacia que favorecerán una transición más armoniosa y menos estresante hacia el retiro.

En relación con la desmesurada importancia de la AEJ para manejar las finanzas sobre los indicadores de ajuste y las intenciones de los trabajadores mayores, sólo queda recalcar la importancia de la planificación financiera de la jubilación, que debería ser apoyada por intervenciones específicas en el ámbito laboral. Tomando en cuenta además que esta faceta de la planificación es especialmente importante para los trabajadores de menores ingresos y para las mujeres, parece recomendable que en el diseño de tales intervenciones se tenga especialmente en cuenta a estas poblaciones de riesgo (Taylor y Geldhauser, 2007).

En resumen, el presente trabajo nos deja algunas cuestiones que abren la perspectiva a nuevas investigaciones. En primer lugar, solo es posible establecer de forma fehaciente la influencia de la AEJ sobre el bienestar de las personas jubiladas a través de diseños de tipo longitudinal, por lo tanto, este debería ser el enfoque predominante en los estudios venideros. En segundo lugar, los estudios precedentes han apuntado la importancia que tiene la visión de las pérdidas futuras asociadas a jubilarse en las decisiones que se toman y el bienestar que se obtiene. En este sentido, correspondería investigar las percepciones asociadas al cambio de situación, con sus facetas positivas y negativas para las personas, en orden a ganar una comprensión más amplia de este proceso. 


\section{Referencias}

Alcover, C. M., Topa, G. y Fernández, J. J. (2014). La gestión organizacional de los trabajadores mayores y los procesos de mantenimiento, prolongación y salida de la vida laboral. Papeles del psicólogo, 35(2), 91-98.

Australian Bureau of Statistics. (2012). Retirement and retirement intentions. Recuperado de http:www.abs.gov.au.

Bandura, A. (1997). Self-efficacy: The exercise of control. New York: Freeman.

Campbell, A., Converse, P. E. y Rodgers, W. L. (1976). The quality of American life: Perceptions, evaluations, and satisfactions. New York: Russell Sage Foundation.

De Judicibus, M. A. y McCabe, M. P. (2005). Economic deprivation and its effects on subjective wellbeing in families of people with multiple sclerosis. Journal of Mental Health, 14(1), 49-59.

Diener, E. D., Emmons, R. A., Larsen, R. J.y Griffin, S. (1985). The satisfaction with life scale. Journal of Personality Assessment, 49(1), 71-75.

Feldman, D. C. (2013). Feeling like it's time to retire: A fit perspective on early retirement decisions. En M. Wang (Ed.), The Oxford Handbook of Retirement, (pp. 280-292). Oxford, New York: Oxford University Press.

Floyd, F. J., Haynes, S. N., Doll, E. R. y Winemiller, D. (1992). Assessing retirement satisfaction and perceptions of retirement experiences. Psychology and $\mathrm{Ag}$ ing, 7, 609-621.

Fretz, B. R., Kluge, N. A., Ossana, S. M., Jones, S. M. y Merikangas, M. W. (1989). Intervention targets for reducing preretirement anxiety and depression. Journal of Counselling Psychology, 36, 301-307.

Gallo, W., Bradley, E., Siegel y M. y Kasl, S. (2001). The impact of involuntary job loss on subsequent alcohol consumption by older workers: findings from the Health and Retirement Survey. Journal of Gerontology, 56B, S3-S9.

Hanratty, B., Holland, P., Jacoby, A. y Whitehead, M. (2007). Review article: financial stress and strain associated with terminal cancer -a review of the evidence. Palliative Medicine, 21(7), 595-607.
Harper, M. (2005). Retirement modelling: An exploration of the effects of retirement role model characteristics on retirement self-efficacy and life satisfaction in midlife workers. Dissertation Abstracts International UMI Nr: 3186005.

Himmelstein, D., U., Warren, E., Thorne, D. y Woolhandler, S. (2005). Market Watch: Illness and Injury as contributors to bankruptcy. Health Affairs. Disponible en: http://content.healthaffairs.org/content/early/2005/02/ 02/hlthaff.w5.63.full.pdf + html

López, E. (1998). Tratamiento de la colinealidad en regresión múltiple. Psicothema, 10(2), 491-507.

Neuhs, H. P. (1990). Retirement self-efficacy: The effects of socioeconomic status, life satisfaction, health, and readiness for retirement. Journal of New York State Nurses Association, 21, 15-20.

Oh, I., Guay, R. P., Kim, K., Harold, C., Lee, J., Heo, Ch. y Shin, K. (2013). Fit happens globally: a Metaanalytic comparison of the relationships of PersonEnvironment fit dimensions with work attitudes and Performance across East Asia, Europe, and North America. Personnel Psychology, 67(1), 99-152.

Peila-Shuster, J. J. (2011). Retirement Self - efficacy: the effects of a Pre-retirement strengths - based intervention on retirement Self-efficacy and an exploration of the relationships between positive affect and retirement self-efficacy(Tesis doctoral, Colorado State University).

Potočnik, K., Tordera, N. y Peiró, J. M. (2009). The role of human resource practices and group norms in the retirement process. European Psychologist, 14(3), 193.

Salanova, M., Peiró, J. M. y Schaufeli, W. B. (2002). Self-efficacy specificity and burnout among information technology workers: An extension of the job demand-control model. European Journal of Work and Organizational Psychology, 11(1), 1-25.

Shultz, K. S., y Adams, G. A. (Eds.). (2007). Aging and work in the 21st century. Mahwah, NJ: Psychology Press.

Stajovic, A. D. y Luthans, F. (1998). Self-efficacy and work-related performance: A metaanalysis. Psychological Bulletin, 124, 240-261. 
Topa, G. y Alcover, C. M. (2015). Psychosocial factors in retirement intentions and adjustment: A multisample study. Career Development International.

Topa, G., Moriano, J. A., Depolo, M., Alcover, C. y Morales, J. (2009). Antecedents and consequences of retirement planning and decision-making: A metaanalysis and model. Journal of Vocational Behavior, $75,38-55$.

Topa, G., Moriano, J., Depolo, M., Alcover, C. y Moreno, A. (2011). Retirement and Wealth relationships: Meta-analysis and SEM. Research on Aging, 33(5), 501-528.

Valero, E., y Topa, G. (2014). Brief Retirement SelfEfficacy-11 Scale (BRSE-11) Factorial Structure and Validity. Journal of Career Assessment, 1069072714553567.

Zaniboni, S., Sarchielli, G. y Fraccaroli, F. (2010). How are psychosocial factors related to retirement intentions? International Journal of Manpower, 31(3), 271-285.

Zappalà, S., Depolo, M., Fraccaroli, F., Guglielmi, D. y Sarchielli, G. (2008). Postponing job retirement? Psychosocial influences on the preference for early or late retirement. Career Development International, 13(2), 150-167. 